\title{
Eigenvalue enclosures and exclosures for non-self-adjoint problems in hydrodynamics
}

\author{
B. Malcolm Brown, Matthias Langer, Marco Marletta, Christiane Tretter and \\ Markus Wagenhofer
}

\begin{abstract}
In this paper we present computer-assisted proofs of a number of results in theoretical fluid dynamics and in quantum mechanics. An algorithm based on interval arithmetic yields provably correct eigenvalue enclosures and exclosures for non-self-adjoint boundary eigenvalue problems, the eigenvalues of which are highly sensitive to perturbations. We apply the algorithm to: the Orr-Sommerfeld equation with Poiseuille profile to prove the existence of an eigenvalue in the classically unstable region for Reynolds number $R=5772.221818$; the Orr-Sommerfeld equation with Couette profile to prove upper bounds for the imaginary parts of all eigenvalues for fixed $R$ and wave number $\alpha$; the problem of natural oscillations of an incompressible inviscid fluid in the neighbourhood of an elliptical flow to obtain information about the unstable part of the spectrum off the imaginary axis; Squire's problem from hydrodynamics; and resonances of one-dimensional Schrödinger operators.
\end{abstract}

\section{Introduction}

The behaviour and stability of many physical systems are connected with the spectral properties of non-self-adjoint operators. However, numerical approximations of eigenvalues of non-selfadjoint operators (even matrices) may fail dramatically. For example, the non-normal $7 \times 7$ matrix

$$
\left(\begin{array}{rrrrrrr}
289 & 2054 & 326 & 128 & 70 & 32 & 6 \\
1152 & 30 & 1312 & 512 & 288 & 128 & 32 \\
-29 & -1990 & 766 & 384 & 1018 & 224 & 58 \\
512 & 128 & 640 & 0 & 640 & 512 & 128 \\
1053 & 2246 & -514 & -384 & -766 & 800 & 198 \\
-287 & -6 & 1722 & -128 & 1978 & -30 & -2042 \\
-2176 & -285 & -1563 & -512 & -539 & -1152 & -287
\end{array}\right),
$$

going back to Godunov (see [7]), has the eigenvalues

$$
0,1,1, \pm 2, \pm 4 \text {, }
$$

which are all real. However, no matter what software is used, numerical computations yield a set of complex eigenvalues, such as

$$
8.57 \pm 3.73 i, 2.29 \pm 8.33 i,-5.43 \pm 6.56 i,-8.85
$$

with imaginary parts as large as 8.33, which are nowhere near the true eigenvalues (see [10, p. 547] and a similar example in [26, p. 489]). The reason for this is that owing to the nonnormality of the matrix, its eigenvalues are highly sensitive to perturbations, and therefore unavoidable rounding errors render the numerical eigenvalue computations unreliable.

This example shows that there is a need for computer-assisted proofs if we want to be sure that a numerically computed eigenvalue of a non-self-adjoint operator is indeed close to a

Received 22 December 2008; revised 6 May 2009.

2000 Mathematics Subject Classification 65L15, 65G30 (primary), 76E99 (secondary). 
true eigenvalue. It is the aim of this paper to develop such proofs by means of a combination of interval arithmetic calculations and analytic methods such as the argument principle for counting eigenvalues in boxes.

In [1], computer-assisted proofs of this kind were established for singular second-order SturmLiouville problems with complex potentials. In the present paper we develop an approach that allows us to treat a greater variety of non-self-adjoint eigenvalue problems. They include fourthorder problems such as the Orr-Sommerfeld equation (with Poiseuille or Couette profile), a parameterised family of systems of first-order ordinary differential equations arising in the study of elliptical vortices in hydrodynamics after separation of variables, and further second-order problems such as Squire's equation and resonances of one-dimensional Schrödinger operators.

Briefly, our method for obtaining provably correct eigenvalue enclosures and exclosures consists of several steps. First, eigenvalues are localised numerically by a floating point approximation. For eigenvalue enclosures, an algorithm based on interval arithmetic and the argument principle is then used to prove that a small box around the approximate eigenvalue contains exactly one true eigenvalue of the given problem. For eigenvalue exclosures, additional analytical information about the location of the spectrum is required that allows us to reduce the eigenvalue exclosure to a compact subset of the complex plane.

We apply the method developed to three problems from hydrodynamics and to resonance problems in quantum mechanics. For the Orr-Sommerfeld equation with Poiseuille profile, which has been conjectured to be unstable for sufficiently large Reynolds numbers since the $\mathrm{PhD}$ thesis of Heisenberg from 1922 (see [9]), we enclose an eigenvalue in the classically unstable half-plane for Reynolds number $R=5772.221818$ (Subsection 3.1). In addition, we prove that for selected $R$ and wave numbers $\alpha$, all eigenvalues lie in the classically stable half-plane $\Im(\lambda)<0$. For the Orr-Sommerfeld equation with Couette profile, which has been known to be classically stable since the work of Romanov in 1971 (see [21]), we establish an explicit negative upper bound for the imaginary parts of the eigenvalues for selected $R$ and wave numbers $\alpha$ (Subsection 3.2). Here and throughout this paper, classical stability means that there are no eigenvalues giving rise to exponential growth of perturbations. Of course, it is important to observe that in situations such as the Orr-Sommerfeld problem where the underlying operators are highly non-normal, other mechanisms such as large transient growth mean that, in the laboratory, instability is already observed at Reynolds numbers which are much lower than the classical stability limit; see [27]. In particular, the classically stable Couette flow is observed to be unstable at sufficiently high Reynolds numbers.

In Section 4, we consider the problem of natural oscillations of an incompressible inviscid fluid in the neighbourhood of an elliptical flow, which arises in the stability theory of elliptical vortices. Although the spectrum is continuous here, separation of variables permits us to describe it as the union of the point spectra of a parameterised family of eigenvalue problems to which our algorithm applies. Analytically, it is known that if the flow is circular, then the spectrum lies on the imaginary axis; in the non-circular case, however, the spectrum consists of the imaginary axis plus infinitely many segments parallel to the real axis that intersect the imaginary axis at all integers and maybe also at half-integers (see $[3, \mathbf{1 5}])$, and thus the flow is certainly unstable. The width of these segments, which depends on the ellipticity parameter, is not known analytically. By means of our interval arithmetic-based method, we establish explicit upper bounds for this width and hence upper and lower bounds for the real part of the spectrum.

Finally, in Section 5, we apply our method to two second-order problems: Squire's problem from hydrodynamics and the resonance problem for one-dimensional Schrödinger operators. These examples illustrate that the algorithm developed in the present paper is not only more widely applicable but also performs better than the one established in [1].

Before proceeding, there are two issues on which we wish to comment.

Firstly, the case for validated numerical computations and computer-assisted proofs is still a matter of some controversy in the scientific computing community. We do not claim in this 
article that validated computations are needed in every situation: however, we draw the reader's attention to the figures in $[4$, p. 417], which show that a very carefully designed numerical method may give misleading results simply because of the limitations of computer arithmetic, even in double precision.

Secondly, we emphasise that the approach which we have taken here, based on shooting for ODEs, is not the only one possible. Plum et al. $[\mathbf{1 2}, \mathbf{1 3}, \mathbf{2 8}]$ have developed very effective approaches based on variational methods and fixed-point theorems. The advantage of our approach is that it is possible to establish eigenvalue exclosures: regions of the complex plane guaranteed to contain no eigenvalues. It is also possible to count eigenvalues in a large region without actually finding them, and multiple eigenvalues need not pose a problem. The advantage of the approach of Plum et al. is that it generalises to PDEs.

\section{Outline of the algorithm}

In this section we describe an algorithm for obtaining guaranteed eigenvalue enclosures and exclosures as well as eigenvalue counts in rectangles of the complex plane. This algorithm combines an interval arithmetic-based code for solving ordinary differential equations with the argument principle for counting zeros of analytic functions.

We study boundary eigenvalue problems for ordinary differential equations with separated boundary conditions on a compact interval $\left[t_{0}, t_{1}\right] \subset \mathbb{R}$. First, we find solutions of the corresponding first-order system with several linearly independent initial values at $t_{0}$ that all satisfy the given boundary conditions in $t_{0}$. A point $\lambda \in \mathbb{C}$ is an eigenvalue if there exists a non-trivial linear combination of these solutions satisfying the boundary conditions in $t_{1}$. For the numerical implementation, all these different initial value problems are put together in one equivalent bigger system,

$$
y^{\prime}=f(y, \lambda), \quad y\left(t_{0}, \lambda\right)=y_{0}
$$

of size $n$, say. The solution $y(\cdot, \lambda)$ is a $\mathbb{C}^{n}$-valued function on $\left[t_{0}, t_{1}\right]$ that depends analytically on the eigenvalue parameter $\lambda$. The problem of matching the original boundary conditions in $t_{1}$, and hence the eigenvalue problem, can now be formulated as a scalar (determinant-like) equation of the form

$$
g(\lambda):=h\left(y\left(t_{1}, \lambda\right)\right)=0
$$

with a function $h: \mathbb{C}^{n} \rightarrow \mathbb{C}$. Results for the original boundary eigenvalue problem with guaranteed error bounds are obtained by:

(i) solving the initial value problem (2.1) with an interval arithmetic-based code;

(ii) localising the zeros of the scalar analytic function $g$ in (2.2) with an interval-valued version of the argument principle.

For (i) we employ the interval arithmetic-based software library VNODE developed by Nedialkov, Jackson and Pryce (see [18]). In interval arithmetic, all operations are performed with complex intervals $[z]=[x]+[y] i$ where $[x],[y] \subset \mathbb{R}$ are closed intervals or singletons. The code VNODE applies to systems (2.1) where the eigenvalue parameter $\lambda$ is replaced by a complex interval $[\lambda]$ and the initial value $y_{0}$ by a complex interval-valued vector $\left[y_{0}\right]=\left[y_{0,1}\right] \times \ldots \times\left[y_{0, n}\right]$, and where $f$ is a composition of elementary functions implemented in interval arithmetic. For every $t \in\left[t_{0}, t_{1}\right]$, the code calculates an interval vector $[y(t,[\lambda])]$ such that for every solution $y$ of (2.1) with $y_{0} \in\left[y_{0}\right]$ and $\lambda \in[\lambda]$, the guaranteed enclosure $y(t, \lambda) \in[y(t,[\lambda])]$ holds.

For (ii) we use the enclosure for $t=t_{1}$ from (i) to obtain guaranteed enclosures for $g([\lambda])$ for given complex intervals $[\lambda]$. The following version of the argument principle allows us to determine the number of zeros of the analytic function $g$ in a given rectangle; see [1, Lemma 3.5]. 
Lemma 2.1. Let $\mathcal{R} \subset \mathbb{C}$ be a closed rectangle with sides parallel to the axes, let $\left(\mu_{j}\right)_{j=0}^{N}$ be a sequence of points on the boundary $\partial \mathcal{R}$ including the corners of $\mathcal{R}$, ordered counter-clockwise with $\mu_{N}=\mu_{0}$, and let $g$ be an analytic function defined on a neighbourhood of $\mathcal{R}$ such that $g\left(\mu_{j}\right) \neq 0$ for $j=0,1, \ldots, N-1$. If

$$
\Re\left(\frac{g\left(\left[\ell_{j, j+1}\right]\right)}{g\left(\mu_{j}\right)}\right)>0 \quad \text { for } j=0,1, \ldots, N-1,
$$

where $\left[\ell_{j, j+1}\right]$ denotes the line segment from $\mu_{j}$ to $\mu_{j+1}$, then the number of zeros of $g$ in the interior of $\mathcal{R}$ equals

$$
\frac{1}{2 \pi} \sum_{j=0}^{N-1} \operatorname{Arg}\left(\frac{g\left(\mu_{j+1}\right)}{g\left(\mu_{j}\right)}\right),
$$

and $g$ has no zeros on the boundary $\partial \mathcal{R}$; here $\operatorname{Arg} z$ denotes the principal argument of $z$, that is, the argument of $z$ such that $-\pi<\operatorname{Arg} z \leqslant \pi$ for $z \neq 0$.

Sketch of the algorithm. The algorithm consists of the following four steps.

Step 1. Choose a rectangle $\mathcal{R}$ and a sequence of points $\left(\mu_{j}\right)_{j=0}^{N}$ on $\partial \mathcal{R}$ as in Lemma 2.1.

Step 2. For $j=0,1, \ldots, N-1$, calculate an enclosure for the quotient $g\left(\left[\ell_{j, j+1}\right]\right) / g\left(\mu_{j}\right)$ in (2.3) using the code VNODE, as outlined in (i).

Step 3. If this enclosure is contained in the open right half-plane, proceed to Step 4; otherwise, go back to Step 1 and make a new choice of $\mathcal{R}$ and/or $\left(\mu_{j}\right)_{j=0}^{N}$.

Step 4. Calculate enclosures for (2.4) again using (i); if an enclosure contains exactly one integer, say $k$, then the original eigenvalue problem has exactly $k$ eigenvalues in the interior of $\mathcal{R}$ and none on the boundary $\partial \mathcal{R}$.

The algorithm yields an eigenvalue count for the rectangle $\mathcal{R}$; in particular, if $k=0$, we obtain an eigenvalue exclosure. In order to enclose a single eigenvalue to a certain accuracy, we subdivide the rectangle $\mathcal{R}$ into smaller rectangles and rerun the algorithm.

In practice, difficulties may arise if the eigenvalue is close to the boundary of the box $\mathcal{R}$, as it may then become extremely difficult to compute sets containing $g\left(\left[\ell_{j, j+1}\right]\right) / g\left(\mu_{j}\right)$ that are in the right half-plane; see [1, Remark 3.6] for suggestions on how to overcome these problems.

Notation. Throughout this paper, we use an abbreviation for real intervals: for example, $0.2378_{13}^{41}$ denotes the interval [0.237813, 0.237841$]$.

\section{Eigenvalue enclosures and exclosures for the Orr-Sommerfeld equation}

In this section we present a method for reliably enclosing the eigenvalues of the Orr-Sommerfeld operator pencil, which arises in a linear stability analysis of flow of an incompressible viscous fluid between two infinite parallel planes. The problem consists of the equation

$$
\left(\left(-\mathrm{D}^{2}+\alpha^{2}\right)^{2}+i \alpha R\left(V\left(-\mathrm{D}^{2}+\alpha^{2}\right)+V^{\prime \prime}\right)\right) y=i \alpha R c\left(-\mathrm{D}^{2}+\alpha^{2}\right) y
$$

on the interval $[-1,1]$, with boundary conditions

$$
y( \pm 1)=y^{\prime}( \pm 1)=0 .
$$

Here $\mathrm{D}:=d / d x$ stands for the derivative, $\alpha \in \mathbb{R}$ is a wave number, $R \geqslant 0$ is the Reynolds number, $V$ is a real-valued function representing the undisturbed stream velocity, and the wave speed $c$ is the spectral parameter (see [5, Section 3.2; 16, Section 25]).

The Orr-Sommerfeld equation (3.1) arises from linearisation of the Navier-Stokes equations if two-dimensional perturbations $(v, 0, w)$ of the velocity profile $(0,0, V)$ are considered. Because of the divergence-free condition, the functions $v$ and $w$ can be written as $v=\partial \psi / \partial z$ and $w=-\partial \psi / \partial x$ where $\psi$ is the so-called stream function, which is then factorised as

$$
\psi(x, z, t)=y(x) e^{i \alpha(z-c t)} .
$$


The case where $\Im(c)<0$ corresponds to classical linear stability, while $\Im(c)>0$ corresponds to classical linear instability of the perturbation (see $[\mathbf{1 6}, \mathbf{2 2}])$. In the classical linear theory, information is sought about the so-called neutral stability curve in the $(R, \alpha)$-plane, which separates domains where the basic motion is linearly stable (that is, $\Im(c)<0$ for all eigenvalues c) from those where it is linearly unstable (that is, $\Im(c)>0$ for at least one eigenvalue $c$ ); see [16, Section 3.2].

In the following, we consider the two standard flow profiles: plane Poiseuille flow where $V(x)=1-x^{2}$ (see Subsection 3.1) and plane Couette flow where $V(x)=x$ (see Subsection 3.2).

First of all, we transform the fourth-order boundary eigenvalue problem (3.1)-(3.2) to a first-order system. To this end, we substitute

$$
u_{1}:=y, \quad u_{2}:=y^{\prime}, \quad u_{3}:=\left(\mathrm{D}^{2}-\alpha^{2}\right) y, \quad u_{4}:=\left(\mathrm{D}^{2}-\alpha^{2}\right) \frac{y^{\prime}}{\sqrt{R}} .
$$

Then (3.1) is equivalent to the first-order system

$$
\left(\begin{array}{l}
u_{1} \\
u_{2} \\
u_{3} \\
u_{4}
\end{array}\right)^{\prime}=\left(\begin{array}{cccc}
0 & 1 & 0 & 0 \\
\alpha^{2} & 0 & 1 & 0 \\
0 & 0 & 0 & \sqrt{R} \\
-i \alpha \sqrt{R} V^{\prime \prime} & 0 & \frac{\alpha^{2}+i \alpha R(V-c)}{\sqrt{R}} & 0
\end{array}\right)\left(\begin{array}{l}
u_{1} \\
u_{2} \\
u_{3} \\
u_{4}
\end{array}\right)
$$

Let

$$
u^{ \pm}(\cdot, c):=\left(\begin{array}{cc}
u_{11}^{ \pm} & u_{12}^{ \pm} \\
u_{21}^{ \pm} & u_{22}^{ \pm} \\
u_{31}^{ \pm} & u_{32}^{ \pm} \\
u_{41}^{ \pm} & u_{42}^{ \pm}
\end{array}\right)(\cdot, c)
$$

be solution matrices of (3.3) such that

$$
u^{-}(-1, c)=\left(\begin{array}{cc}
0 & 0 \\
0 & 0 \\
1 & 0 \\
0 & 1
\end{array}\right), \quad u^{+}(1, c)=\left(\begin{array}{cc}
0 & 0 \\
0 & 0 \\
1 & 0 \\
0 & 1
\end{array}\right)
$$

The following lemma is easy to prove.

Lemma 3.1. Let $\xi \in[-1,1]$ be fixed. The complex number $c$ is an eigenvalue of the Orr-Sommerfeld equation if and only if

$$
f_{\xi}(c):=\operatorname{det}\left(u^{-}(\xi, c) \quad u^{+}(\xi, c)\right)=0
$$

Unsurprisingly, it turns out that solving (3.3) directly is a bad idea: it is neither fast to solve nor does it permit large Reynolds numbers $R$.

Instead, we shall use the compound matrix method (see, for example, [8, Section 2.1] and the references given there). Let the functions $z_{j}^{ \pm}(\cdot, c), j=1, \ldots, 6$, be defined by

$$
z_{1}^{ \pm}(\cdot, c):=\operatorname{det}\left(\begin{array}{cc}
u_{11}^{ \pm} & u_{12}^{ \pm} \\
u_{21}^{ \pm} & u_{22}^{ \pm}
\end{array}\right)(\cdot, c), \quad z_{2}^{ \pm}(\cdot, c):=\operatorname{det}\left(\begin{array}{cc}
u_{11}^{ \pm} & u_{12}^{ \pm} \\
u_{31}^{ \pm} & u_{32}^{ \pm}
\end{array}\right)(\cdot, c),
$$




$$
\begin{aligned}
& z_{3}^{ \pm}(\cdot, c):=\operatorname{det}\left(\begin{array}{cc}
u_{11}^{ \pm} & u_{12}^{ \pm} \\
u_{41}^{ \pm} & u_{42}^{ \pm}
\end{array}\right)(\cdot, c), \quad z_{4}^{ \pm}(\cdot, c):=\operatorname{det}\left(\begin{array}{cc}
u_{21}^{ \pm} & u_{22}^{ \pm} \\
u_{31}^{ \pm} & u_{32}^{ \pm}
\end{array}\right)(\cdot, c), \\
& z_{5}^{ \pm}(\cdot, c):=\operatorname{det}\left(\begin{array}{ll}
u_{21}^{ \pm} & u_{22}^{ \pm} \\
u_{41}^{ \pm} & u_{42}^{ \pm}
\end{array}\right)(\cdot, c), \quad z_{6}^{ \pm}(\cdot, c):=\operatorname{det}\left(\begin{array}{ll}
u_{31}^{ \pm} & u_{32}^{ \pm} \\
u_{41}^{ \pm} & u_{42}^{ \pm}
\end{array}\right)(\cdot, c) .
\end{aligned}
$$

These functions satisfy the differential equations

$$
\begin{aligned}
& z_{1}^{\prime}(\cdot, c)=z_{2}(\cdot, c) \\
& z_{2}^{\prime}(\cdot, c)=\sqrt{R} z_{3}(\cdot, c)+z_{4}(\cdot, c) \\
& z_{3}^{\prime}(\cdot, c)=\frac{\alpha^{2}+i \alpha R(V-c)}{\sqrt{R}} z_{2}(\cdot, c)+z_{5}(\cdot, c) \\
& z_{4}^{\prime}(\cdot, c)=\alpha^{2} z_{2}(\cdot, c)+\sqrt{R} z_{5}(\cdot, c), \\
& z_{5}^{\prime}(\cdot, c)=i \alpha \sqrt{R} V^{\prime \prime} z_{1}(\cdot, c)+\alpha^{2} z_{3}(\cdot, c)+\frac{\alpha^{2}+i \alpha R(V-c)}{\sqrt{R}} z_{4}(\cdot, c)+z_{6}(\cdot, c), \\
& z_{6}^{\prime}(\cdot, c)=i \alpha \sqrt{R} V^{\prime \prime} z_{2}(\cdot, c)
\end{aligned}
$$

and the initial conditions

$$
z_{1}^{ \pm}( \pm 1, c)=z_{2}^{ \pm}( \pm 1, c)=z_{3}^{ \pm}( \pm 1, c)=z_{4}^{ \pm}( \pm 1, c)=z_{5}^{ \pm}( \pm 1, c)=0, \quad z_{6}^{ \pm}( \pm 1, c)=1 .
$$

Using Laplace expansion, we obtain

$$
f_{\xi}(c)=\left(z_{1}^{-} z_{6}^{+}-z_{2}^{-} z_{5}^{+}+z_{3}^{-} z_{4}^{+}+z_{4}^{-} z_{3}^{+}-z_{5}^{-} z_{2}^{+}+z_{6}^{-} z_{1}^{+}\right)(\xi, c) .
$$

According to Lemma 3.1, we have to find the zeros of $f_{\xi}$. To this end, for fixed $\xi$, we apply the argument principle described in Lemma 2.1 to the function $f_{\xi}$.

REMARK 3.2. Note that for a symmetric potential such that $V(x)=V(-x)$ for $x \in[-1,1]$, it is sufficient to calculate, for instance, $z_{j}^{-}(0, c)$ for $j=1, \ldots, 6$. In this case, $f_{0}$ simplifies to

$$
f_{0}(c)=\left(z_{1}^{-} z_{6}^{-}+z_{2}^{-} z_{5}^{-}+z_{3}^{-} z_{4}^{-}+z_{4}^{-} z_{3}^{-}+z_{5}^{-} z_{2}^{-}+z_{6}^{-} z_{1}^{-}\right)(0, c) .
$$

\subsection{Plane Poiseuille flow}

In this subsection we consider the case in which the two planes are stationary. For this so-called Poiseuille flow, the unperturbed flow moves more slowly near the bounding planes and faster in the centre of the channel between the planes; here the unperturbed velocity profile $V$ is of the form $V(x)=1-x^{2}, x \in[-1,1]$. In this case, the neutral stability curve in the $(R, \alpha)$-plane is also known as Heisenberg's tongue (see [6, p. 35, Figure 1.1.2]); the critical Reynolds number is the $R$-coordinate of the leftmost point of this curve. So far, only numerical calculations for this critical value have been available, which indicate that plane Poiseuille flow becomes unstable at Reynolds numbers $R$ close to 5772.22 ; see, for example, [20, p. 697; 24].

Asymptotic and numerical calculations of the critical Reynolds number for Poiseuille flow have a long history: see [17] for a discussion of the early numerical experiments, [25] for one of the earliest calculations by shooting, and [20] for a calculation which was the first to claim accuracy to two decimal places, reporting that the critical Reynolds number is near $R=5772.22$ and that the first unstable eigenvalue appears for $1.02055 \leqslant \alpha \leqslant 1.02057$. More recent calculations include those of $\mathrm{Ng}$ and Reid [19], using shooting with compound matrices, and of Dongarra, Straughan and Walker [4], who used a high precision Chebychev tau-algorithm coupled with quadruple precision arithmetic. For a review see the books [23] by Schmid and Henningson or [26] by Trefethen and Embree, and references therein.

To show the classical instability of plane Poiseuille flow for a certain Reynolds number $R$, we have to find at least one $\alpha$ and a corresponding eigenvalue enclosure lying completely in 
the open upper half-plane. To show that the basic motion with Poiseuille profile is classically stable for certain values of $R$ and $\alpha$, we have to guarantee that all eigenvalues of (3.1)-(3.2) lie in the open lower half-plane; to this end, we use the following global analytic enclosure for the Poiseuille eigenvalues; see [14, Corollary 5.4].

Proposition 3.3. Let $V(x)=1-x^{2}$ for $x \in[-1,1]$. Then every eigenvalue $c \in \mathbb{C}$ of (3.1)-(3.2) satisfies the estimates

$$
\Im(c)<\frac{1}{\alpha}\left(1-\frac{\alpha^{2}}{R}\right) \leqslant \frac{1}{\alpha}, \quad-\frac{1}{\alpha^{2}} \leqslant \Re(c) \leqslant 1 .
$$

As a consequence, if for a pair $(R, \alpha)$ there is no eigenvalue $c$ in the rectangle

$$
\left[-\frac{1}{\alpha^{2}}, 1\right]+\left[0, \frac{1}{\alpha}\right] i
$$

then there is no eigenvalue in the closed upper half-plane and hence the basic motion with Poiseuille profile is classically stable for this pair $(R, \alpha)$.

3.1.1. Enclosure of the critical Reynolds number for fixed $\alpha$. As already mentioned, Orszag [20] reported a critical Reynolds number near $R=5772.22$, with the first unstable eigenvalue appearing for $1.02055 \leqslant \alpha \leqslant 1.02057$.

Starting from pairs $(R, \alpha)$ with $\alpha$ in the range [1.02055, 1.02057], our algorithm gave the guaranteed results shown in Table 1.

It follows that the neutral stability curve in the $(R, \alpha)$-plane passes between the two points

$$
(5772.221817,1.020551) \text { and }(5772.221818,1.020551) \text {, }
$$

that for $R=5772.221818$ an eigenvalue $c$ in the unstable half-plane exists which has

$$
0<\Im(c)<2 \cdot 10^{-12}
$$

and hence that the critical Reynolds number $R_{c}$ satisfies the guaranteed upper estimate

$$
R_{c} \leqslant 5772.221818 \text {. }
$$

Thus we have proved the first guaranteed enclosure for an unstable eigenvalue for a Reynolds number close to the suggested critical value 5772.22 .

Remark 3.4. Simultaneously, but using a different method, Watanabe, Plum and Nakao obtained a guaranteed enclosure for an unstable eigenvalue for the larger Reynolds number $R=5775$ and $\alpha=1.02$; see [28]. The imaginary part of this eigenvalue satisfies $1.06103 \cdot 10^{-6} \leqslant$ $\Im(c) \leqslant 8.0112 \cdot 10^{-6}$ and is thus larger than the unstable eigenvalue that we obtained by a factor of at least $10^{6}$.

3.1.2. Provably classically stable pairs $(R, \alpha)$. Using Proposition 3.3, we were able to prove that in addition to the pair $(5772.221817,1.020551)$ from $\S 3.1 .1$, for the $(R, \alpha)$ pairs in Table 2 there are also no eigenvalues in the closed upper half-plane.

TABLE 1. Enclosures for Poiseuille eigenvalues $c$ with $\Im(c)>0$.

\begin{tabular}{ccc}
\hline$R$ & $\alpha$ & {$[c]$} \\
\hline 5772.221817 & 1.020551 & No eigenvalue with $\Im(c)>0$ \\
5772.221818 & 1.020551 & $0.26400067483_{3}^{5}+0.00000000000_{0}^{2} i$ \\
\hline
\end{tabular}




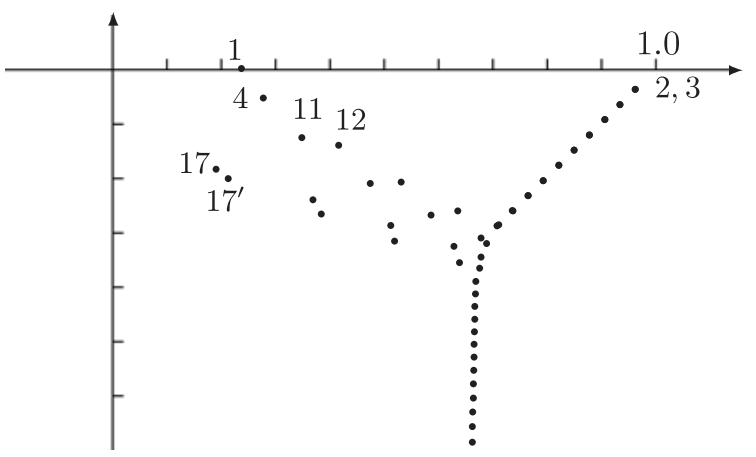

Figure 1. Poiseuille eigenvalues for $R=10000$ and $\alpha=1$.

3.1.3. Eigenvalue enclosures for fixed $(R, \alpha)$. For the values $R=10000$ and $\alpha=1$, we calculated guaranteed enclosures for several eigenvalues that had been found numerically by Orszag; see [20, Table 5]. These eigenvalues were confirmed numerically, and one additional eigenvalue was found, by Dongarra, Straughan and Walker in [4, Section 3]. Here we select some of these numerical values and give the first proof that they do approximate true eigenvalues. The corresponding eigenvalue enclosures are listed in Table 3, in descending order of imaginary part, according to the enumeration of [20, Table 5]; the additional eigenvalue found in $[\mathbf{4},(3.2)]$ is denoted by $17^{\prime}$ (see also Figure 1 ).

In addition, we have been able to show that there are no eigenvalues in the rectangle $[-1,0]+[-1,1] i$ in the left half-plane and that there are exactly three eigenvalues in the rectangle $[0,1]+[-0.04,1] i$ in the right half-plane. Hence, apart from eigenvalue no. 1 in Table 3 , there must be two other eigenvalues in $[0,1]+[-0.04,1] i$.

TABle 2. Parameter pairs $(R, \alpha)$ for which plane Poiseuille flow is classically stable.

\begin{tabular}{cc}
\hline$R$ & $\alpha$ \\
\hline 1000 & 1.0 \\
2000 & 1.0 \\
3000 & 1.0 \\
4000 & 1.0 \\
5000 & 1.0 \\
6000 & 0.5 \\
6000 & 2.0 \\
7000 & 0.5 \\
7000 & 2.0 \\
\hline
\end{tabular}

TABLE 3. Enclosures for Poiseuille eigenvalues for $R=10000$ and $\alpha=1$.

\begin{tabular}{ccc}
\hline & $c$ in $[\mathbf{2 0}]$ (and [4]) & Guaranteed enclosure for $c$ \\
\hline 1 & $0.23752649+0.00373967 i$ & $0.237526488_{76}^{84}+0.003739670_{60}^{71} i$ \\
4 & $0.27720434-0.05089873 i$ & $0.277204343_{74}^{83}-0.050898727_{18}^{27} i$ \\
11 & $0.34910682-0.12450198 i$ & $0.3491068201_{09}^{41}-0.1245019775_{29}^{76} i$ \\
12 & $0.41635102-0.13822652 i$ & $0.416351015_{54}^{61}-0.138226525_{27}^{33} i$ \\
17 & $0.1900592-0.1828219 i$ & $0.190059249_{31}^{42}-0.182821925_{39}^{49} i$ \\
$17^{\prime}$ & $0.21272578-0.19936069 i$ & $0.212725782_{18}^{51}-0.19936069_{46}^{51} i$ \\
\hline
\end{tabular}


REMARK 3.5. The numerical calculations for eigenvalues no. 2 and no. 3 in [20] suggest that these two eigenvalues are very close (with the distance between them on the order of $2.2 \cdot 10^{-5}$ ). Although we were able to find two rectangles around these numerical values such that the enclosure for the expression in (2.4) contains 1 as the only integer, we could not verify condition (2.3) in reasonable time. In Table 4 the corresponding non-rigorous enclosures are marked by [!] .

\subsection{Plane Couette flow}

In this subsection we study the case in which the two planes move in opposite directions. For this so-called Couette flow, the unperturbed flow is completely stationary in the middle of the channel between the two planes and the unperturbed velocity profile $V$ has the form $V(x)=x$, $x \in[-1,1]$. It is known that Couette flow is classically stable at all Reynolds numbers. However, Romanov [21] only proved that there exists a constant $d>0$ such that for each eigenvalue $c$ the estimate $\Im(c) \leqslant-d / R$ holds; no explicit upper bounds for the imaginary parts of the eigenvalues seem to be known at present.

It is the aim of this section to establish such explicit upper bounds using provably correct eigenvalue exclosures. Since only bounded regions can be covered by such a technique, we first employ a result which yields a global explicit bound for the real parts of all eigenvalues of the Orr-Sommerfeld equation with Couette profile.

Proposition 3.6. Let $V(x)=x$ for $x \in[-1,1]$. Then every eigenvalue $c \in \mathbb{C}$ of $(3.1)-(3.2)$ satisfies the estimates

$$
\Im(c)<0, \quad-1 \leqslant \Re(c) \leqslant 1 .
$$

Proof. The estimate for the imaginary part was proved in [21]; more exactly, it is shown there that there is a constant $d>0$ such that $\Im(c) \leqslant-d / R$. The estimate for the real part follows from [14, Corollary 5.4]; see also [11].

3.2.1. Guaranteed upper bounds for the imaginary parts of all eigenvalues for selected pairs $(R, \alpha)$. By Proposition 3.6, if for some $\nu>0$ the rectangle

$$
[-1,1]+[-\nu, 0] i
$$

contains no eigenvalue, then $\Im(c) \leqslant-\nu$ for all Couette eigenvalues $c$. Corresponding guaranteed upper bounds are listed in Table 5 for various $(R, \alpha)$ pairs; their dependence on the Reynolds number is displayed in Figure 2 (the lines therein connect upper bounds belonging to the same value of $\alpha$ ).

3.2.2. Eigenvalue enclosures for fixed $(R, \alpha)$. For $R=13000$ and $\alpha=1$, we calculated guaranteed enclosures for three eigenvalue pairs that were found numerically by Dongarra,

TABLE 4. Non-rigorous enclosures for Poiseuille eigenvalues for $R=10000$ and $\alpha=1$.

\begin{tabular}{ccc}
\hline & $c$ in $[\mathbf{2 0}]$ & Non-rigorous enclosure for $c$ \\
\hline 2 & $0.96463092-0.03516728 i$ & $0.964630915_{31}^{63}-0.035167277_{49}^{82} i^{[!]}$ \\
3 & $0.96464251-0.03518658 i$ & $0.9646425_{09}^{11}-0.03518658_{36}^{40} i^{[!]}$ \\
\hline
\end{tabular}




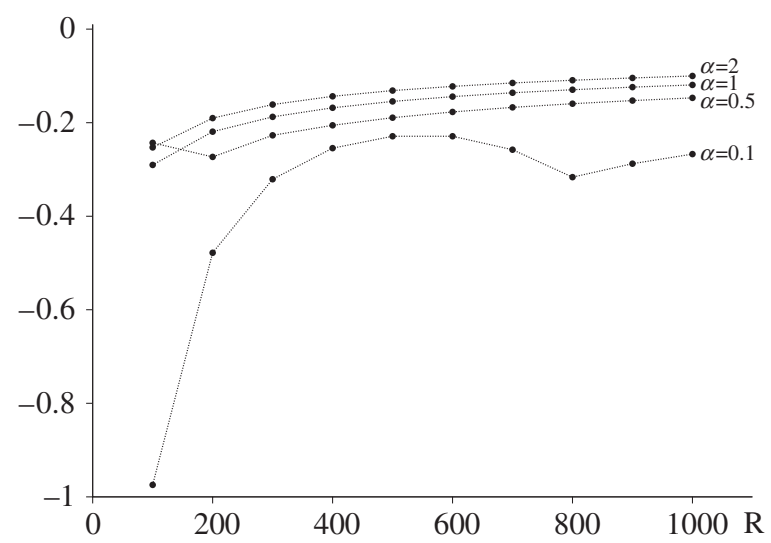

FiguRE 2. Guaranteed upper bounds for the imaginary parts of Couette eigenvalues for $\alpha=0.1,0.5,1$ and 2 .

Straughan and Walker; see [4, Table 4]. Additionally, we were able to guarantee that apart from the first pair in Table 6, there is no other eigenvalue with imaginary part greater than -0.08 .

\section{Elliptical vortices in hydrodynamics}

The linear stability analysis of natural oscillations of an incompressible inviscid fluid in the neighbourhood of an elliptical flow leads to a spectral problem $\mathfrak{A}_{\delta} f=\lambda f$ for a linear operator $\mathfrak{A}_{\delta}$ of the form

$$
\mathfrak{A}_{\delta}=\left(\begin{array}{cc}
\frac{\partial}{\partial \psi} & 0 \\
0 & \frac{\partial}{\partial \psi}
\end{array}\right)+2 N(\rho, \psi)
$$

TABLE 5. Guaranteed upper bounds for the imaginary parts of Couette eigenvalues.

\begin{tabular}{ccccc}
\hline$R$ & $\alpha=0.1$ & $\alpha=0.5$ & $\alpha=1$ & $\alpha=2$ \\
\hline 100 & -0.9742 & -0.2429 & -0.2904 & -0.2527 \\
200 & -0.4781 & -0.2731 & -0.2192 & -0.1901 \\
300 & -0.3209 & -0.2270 & -0.1875 & -0.1612 \\
400 & -0.2545 & -0.2054 & -0.1680 & -0.1436 \\
500 & -0.2288 & -0.1892 & -0.1543 & -0.1313 \\
600 & -0.2290 & -0.1769 & -0.1441 & -0.1222 \\
700 & -0.2577 & -0.1672 & -0.1360 & -0.1150 \\
800 & -0.3164 & -0.1593 & -0.1294 & -0.1091 \\
900 & -0.2876 & -0.1526 & -0.1239 & -0.1042 \\
1000 & -0.2670 & -0.1468 & -0.1191 & -0.1000 \\
\hline
\end{tabular}

TABLE 6. Guaranteed enclosures for Couette eigenvalues for $R=13000$ and $\alpha=1$.

\begin{tabular}{cc}
\hline$c$ in $[4]$ & Guaranteed enclosure of $c$ \\
\hline $\pm 0.8267152337-0.04751548439 i$ & $\pm 0.82761523365_{62}^{94}-0.0475154843_{87}^{91} i$ \\
$\pm 0.7318167785-0.1091860424 i$ & $\pm 0.73181677846_{20}^{36}-0.10918604238_{30}^{46} i$ \\
$\pm 0.8694486153-0.1279149536 i$ & $\pm 0.869448615321_{720}^{991}-0.127914953560_{190}^{451} i$ \\
\hline
\end{tabular}


(see [15]) in the product $\left(L^{2}((0, \infty) \times(0,2 \pi), \rho d \rho d \psi)\right)^{2}$ of weighted $L^{2}$-spaces, with

$$
\begin{aligned}
& N(\rho, \psi)=\left(\begin{array}{cc}
0 & -\frac{1-\delta^{2}}{\rho^{2}(1-\delta \cos (2 \psi))+1-\delta^{2}} \\
1 & \frac{\delta \rho^{2} \sin (2 \psi)}{\rho^{2}(1-\delta \cos (2 \psi))+1-\delta^{2}}
\end{array}\right), \quad \rho \in[0, \infty), \psi \in[0,2 \pi], \\
& N(\infty, \psi)=\left(\begin{array}{cc}
0 & 0 \\
1 & \frac{\delta \sin (2 \psi)}{1-\delta \cos (2 \psi)}
\end{array}\right), \quad \psi \in[0,2 \pi]
\end{aligned}
$$

and boundary conditions

$$
f(\rho, 0)=f(\rho, 2 \pi) \text { for every } \rho \in[0, \infty] .
$$

Here $\rho \in[0, \infty]$ and $\psi \in[0,2 \pi)$ are polar coordinates, and the ellipticity parameter $\delta \in[0,1)$ characterises the geometry of the flow. For the special case of $\delta=0$, the flow becomes circular; in the limiting case $\delta=1$, it would become a linear shear flow. In this simple analysis, the flow is regarded as stable if there is no spectrum in the open right half-plane.

Based on the fact that the operator $\mathfrak{A}_{\delta}$ contains only derivatives with respect to one variable, it was shown in $[\mathbf{3}]$ that

$$
\sigma\left(\mathfrak{A}_{\delta}\right)=\bigcup_{\rho \in[0, \infty]} \sigma\left(A_{\delta}(\rho)\right)=\bigcup_{\rho \in[0, \infty]} \sigma_{\mathrm{p}}\left(A_{\delta}(\rho)\right),
$$

where the linear operator

$$
A_{\delta}(\rho)=\left(\begin{array}{cc}
\frac{d}{d \psi} & 0 \\
0 & \frac{d}{d \psi}
\end{array}\right)+2 N(\rho, \cdot), \quad \rho \in[0, \infty],
$$

acts in the space $\left(L^{2}(0,2 \pi)\right)^{2}$ with $\rho$-independent domain

$$
D:=D\left(A_{\delta}(\rho)\right)=\left\{f(\rho) \in\left(H^{1}(0,2 \pi)\right)^{2}: f(\rho)(0)=f(\rho)(2 \pi)\right\} .
$$

Using the above description of the spectrum of $\mathfrak{A}_{\delta}$, the following analytic theorem about the structure and location of $\sigma\left(\mathfrak{A}_{\delta}\right)$ was proved in [3, Theorem 3.1].

TheOREM 4.1. The operator $\mathfrak{A}_{\delta}$ has no eigenvalues and there are constants $a_{\delta} \geqslant 0, b_{\delta} \geqslant 0$ and $c_{\delta} \in\left[0, \frac{1}{2}\right]$ such that

$$
\sigma\left(\mathfrak{A}_{\delta}\right)=\bigcup_{k \in \mathbb{Z}}\left(i k+\left[-a_{\delta}, a_{\delta}\right]\right) \cup\left(i\left(k+\frac{1}{2}\right)+\left[-b_{\delta}, b_{\delta}\right]\right) \cup\left(i\left[k-c_{\delta}, k+c_{\delta}\right]\right) .
$$

If $b_{\delta}>0$, then $c_{\delta}=\frac{1}{2}$.

For a circular flow where $\delta=0$, it can be shown analytically that $\sigma\left(\mathfrak{A}_{\delta}\right)=i \mathbb{R}$ (that is, $a_{0}=b_{0}=0$ and $c_{0}=\frac{1}{2}$ in Theorem 4.1). For $\delta>0$, numerical results in [3] and [15] have suggested that in Theorem 4.1, the width $b_{\delta}$ of the segments intersecting the imaginary axis at half-integers is zero and that the width $a_{\delta}$ of the segments intersecting the imaginary axis at integers is strictly increasing with $\delta$; in particular, this would mean that the flow is always unstable as soon as $\delta>0$.

In the following, we want to find provably correct enclosures of the constants occurring in Theorem 4.1; that is, we shall determine real intervals $\left[a_{\delta}\right],\left[b_{\delta}\right]$ and $\left[c_{\delta}\right]$ such that we have the inclusions

$$
a_{\delta} \in\left[a_{\delta}\right], \quad b_{\delta} \in\left[b_{\delta}\right], \quad c_{\delta} \in\left[c_{\delta}\right] .
$$


In [3] it was shown that the eigenvalues of $A_{\delta}(\rho)$ can be characterised as follows: let $Y_{\delta, \rho}:[0,1] \rightarrow M_{2}(\mathbb{R})$ be the fundamental solution matrix of the system

$$
\begin{aligned}
& y_{1}^{\prime}=\frac{2 \pi\left(1-\delta^{2}\right)}{\rho^{2}(1-\delta \cos (4 \pi \psi))+1-\delta^{2}} y_{2}, \\
& y_{2}^{\prime}=-2 \pi\left(2 y_{1}+\frac{\delta \rho^{2} \sin (4 \pi \psi)}{\rho^{2}(1-\delta \cos (4 \pi \psi))+1-\delta^{2}} y_{2}\right)
\end{aligned}
$$

on $[0,1]$ such that $Y_{\delta, \rho}(0)$ is the identity matrix, and let $\beta_{\delta}(\rho):=\operatorname{trace}\left(Y_{\delta, \rho}(1)\right)$. (Note that the original equation from $[3]$ on $[0,2 \pi]$ has been transformed to one on the interval $[0,1]$. This had to be done because VNODE does not allow interval-valued boundaries.) Then $\lambda \in \mathbb{C}$ is an eigenvalue of the operator $A_{\delta}(\rho)$ if and only if

$$
e^{-4 \lambda \pi}-\beta_{\delta}(\rho) e^{-2 \lambda \pi}+1=0 .
$$

There are three cases for the value of $\beta_{\delta}(\rho)$ that are of interest in regard to the location of the eigenvalues of $A_{\delta}(\rho)$.

(1) If $2 \leqslant \beta_{\delta}(\rho)$, then $\lambda$ is an eigenvalue of $A_{\delta}(\rho)$ if and only if

$$
\Re(\lambda)= \pm(2 \pi)^{-1} \log \frac{\beta_{\delta}(\rho)+\sqrt{\beta_{\delta}(\rho)^{2}-4}}{2}, \quad \Im(\lambda) \in \mathbb{Z} .
$$

(2) If $-2<\beta_{\delta}(\rho)<2$, then $\lambda$ is an eigenvalue of $A_{\delta}(\rho)$ if and only if

$$
\Re(\lambda)=0, \quad \Im(\lambda) \in \begin{cases}\mathbb{Z} \pm(2 \pi)^{-1} \arctan \frac{\sqrt{4-\beta_{\delta}(\rho)^{2}}}{\beta_{\delta}(\rho)}, & \beta_{\delta}(\rho)>0 \\ \pm \frac{1}{4}+\mathbb{Z}, & \beta_{\delta}(\rho)=0 \\ \frac{1}{2}+\mathbb{Z} \pm(2 \pi)^{-1} \arctan \frac{\sqrt{4-\beta_{\delta}(\rho)^{2}}}{\beta_{\delta}(\rho)}, & \beta_{\delta}(\rho)<0 .\end{cases}
$$

(3) If $\beta_{\delta}(\rho) \leqslant-2$, then $\lambda$ is an eigenvalue of $A_{\delta}(\rho)$ if and only if

$$
\Re(\lambda)= \pm(2 \pi)^{-1} \log \frac{-\beta_{\delta}(\rho)-\sqrt{\beta_{\delta}(\rho)^{2}-4}}{2}, \quad \Im(\lambda) \in \frac{1}{2}+\mathbb{Z} .
$$

Now let

$$
\beta_{\delta}^{-}:=\min \left\{\beta_{\delta}(\rho): \rho \in[0, \infty]\right\}, \quad \beta_{\delta}^{+}:=\max \left\{\beta_{\delta}(\rho): \rho \in[0, \infty]\right\}
$$

We already know from $[3]$ that $\beta_{\delta}^{+} \geqslant 2$ and $\beta_{\delta}^{-}<2$. Moreover, it is clear that

$$
\begin{aligned}
& a_{\delta}=(2 \pi)^{-1} \log \frac{\beta_{\delta}^{+}+\sqrt{\left(\beta_{\delta}^{+}\right)^{2}-4}}{2}, \\
& b_{\delta}= \begin{cases}0, & -2 \leqslant \beta_{\delta}^{-}, \\
(2 \pi)^{-1} \log \frac{-\beta_{\delta}^{-}-\sqrt{\left(\beta_{\delta}^{-}\right)^{2}-4}}{2}, & \beta_{\delta}^{-}<-2,\end{cases}
\end{aligned}
$$




$$
c_{\delta}= \begin{cases}(2 \pi)^{-1} \arctan \frac{\sqrt{4-\beta_{\delta}(\rho)^{2}}}{\beta_{\delta}(\rho)}, & 0<\beta_{\delta}^{-}<2, \\ \frac{1}{4}, & \beta_{\delta}^{-}=0, \\ \frac{1}{2}-(2 \pi)^{-1} \arctan \frac{\sqrt{4-\beta_{\delta}(\rho)^{2}}}{\beta_{\delta}(\rho)}, & -2<\beta_{\delta}^{-}<0, \\ \frac{1}{2}, & \beta_{\delta}^{-} \leqslant-2 .\end{cases}
$$

Thus, to determine the shape of $\sigma\left(\mathfrak{A}_{\delta}\right)$, it remains to calculate $\beta_{\delta}^{ \pm}$. Given closed bounded intervals $[\delta] \subset[0,1)$ and $[\rho] \subset[0, \infty)$, VNODE enables us to determine a closed bounded interval $\left[\beta_{[\delta]}([\rho])\right]$ such that the inclusion

$$
\beta_{\delta}(\rho) \in\left[\beta_{[\delta]}([\rho])\right] \text { for } \delta \in[\delta], \rho \in[\rho]
$$

holds. In particular, getting an appropriate estimate of

$$
\min \left\{\beta_{\delta}(\rho): \rho \in\left[0, \rho_{0}\right]\right\} \quad \text { or } \quad \max \left\{\beta_{\delta}(\rho): \rho \in\left[0, \rho_{0}\right]\right\} \quad \text { for } \delta \in[\delta],
$$

for some $\rho_{0}>0$, reduces to a problem of choosing $[\delta]$ small enough and dividing $\left[0, \rho_{0}\right]$ into a sufficiently fine partition of sub-intervals $[\rho] \subset\left[0, \rho_{0}\right]$.

The estimation of $\beta_{\delta}$ on the remaining unbounded interval $\left[\rho_{0}, \infty\right]$ can be done as follows: observe that for $\rho \in\left[\rho_{0}, \infty\right]$,

$$
0 \leqslant \frac{1-\delta^{2}}{\rho^{2}(1-\delta \cos (4 \pi \psi))+1-\delta^{2}} \leqslant \frac{1+\delta}{\rho_{0}^{2}+1+\delta}
$$

thus

$$
\frac{1-\delta^{2}}{\rho^{2}(1-\delta \cos (4 \pi \psi))+1-\delta^{2}} \in\left[0, \frac{1+\delta}{\rho_{0}^{2}+1+\delta}\right]=: \widetilde{E}_{\delta}\left(\rho_{0}\right) \subset \mathbb{R}
$$

and

$$
\frac{\delta \sin (4 \pi \psi)}{(1-\delta \cos (4 \pi \psi))+\left(1-\delta^{2}\right) \rho^{-2}} \in \frac{\delta \sin (4 \pi \psi)}{(1-\delta \cos (4 \pi \psi))+\left(1-\delta^{2}\right)\left[0, \rho_{0}^{-2}\right]}=: \widetilde{F}_{\delta}\left(\rho_{0}\right) \subset \mathbb{R}
$$

for $\rho \in\left[\rho_{0}, \infty\right]$. Now, by using computed enclosures $\left[E_{[\delta]}\left(\rho_{0}\right)\right]$ and $\left[F_{[\delta]}\left(\rho_{0}\right)\right]$ such that

$$
\widetilde{E}_{\delta}\left(\rho_{0}\right) \subset\left[E_{[\delta]}\left(\rho_{0}\right)\right] \text { and } \widetilde{F}_{\delta}\left(\rho_{0}\right) \subset\left[F_{[\delta]}\left(\rho_{0}\right)\right] \text { for } \delta \in[\delta]
$$

and solving the interval-valued differential equation

$$
\begin{aligned}
& y_{1}^{\prime}=2 \pi\left[E_{[\delta]}\left(\rho_{0}\right)\right] y_{2}, \\
& y_{2}^{\prime}=-2 \pi\left(2 y_{1}+\left[F_{[\delta]}\left(\rho_{0}\right)\right] y_{2}\right),
\end{aligned}
$$

we arrive at an enclosure $\left[\beta_{[\delta]}^{\infty}\left(\rho_{0}\right)\right]$ such that

$$
\beta_{\delta}(\rho) \in\left[\beta_{[\delta]}^{\infty}\left(\rho_{0}\right)\right] \text { for } \delta \in[\delta], \rho \geqslant \rho_{0} .
$$

EXAmple 4.2. For $[\delta]=[0.2,0.21]$ and $\rho_{0}=10$, we obtain the estimate

$$
\beta_{\delta}(\rho) \in\left[\beta_{[\delta]}^{\infty}\left(\rho_{0}\right)\right]=[-0.24615,2.34774] \quad \text { for } \rho \geqslant \rho_{0}, \delta \in[\delta] .
$$

Moreover, estimating $\beta_{[\delta]}^{ \pm}([\rho])$ on the intervals $\rho=[0,0.01],[0.01,0.02], \ldots,[9.99,10]$ we get $\beta_{\delta}^{-} \in[-2.276,-1.760]$ and $\beta_{\delta}^{+} \in[2.126,3.001]$ for $\delta \in[\delta]$. Thus,

$$
a_{\delta} \in[0.056,0.154], \quad b_{\delta} \in[0,0.083], \quad c_{\delta} \in[0.421,0.5] \quad \text { for } \delta \in[0.2,0.21] .
$$

The graph of the calculated enclosure of $\beta_{\delta}$ on $[0,10]$ is shown in Figure 3 . 


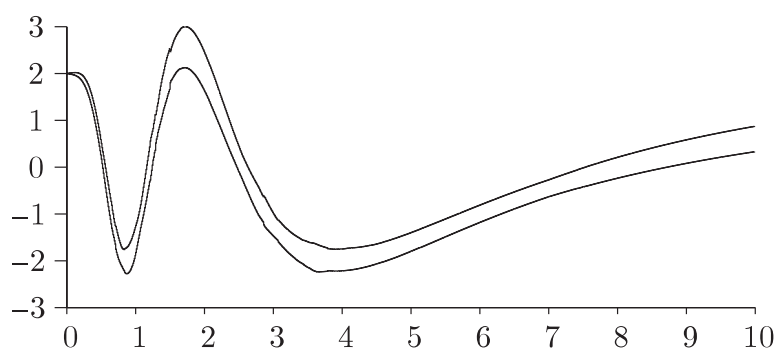

Figure 3. The dependence on $\rho \in[0,10]$ of an enclosure of $\beta_{\delta}(\rho)$ for $\delta \in[\delta]=[0.2,0.21]$.
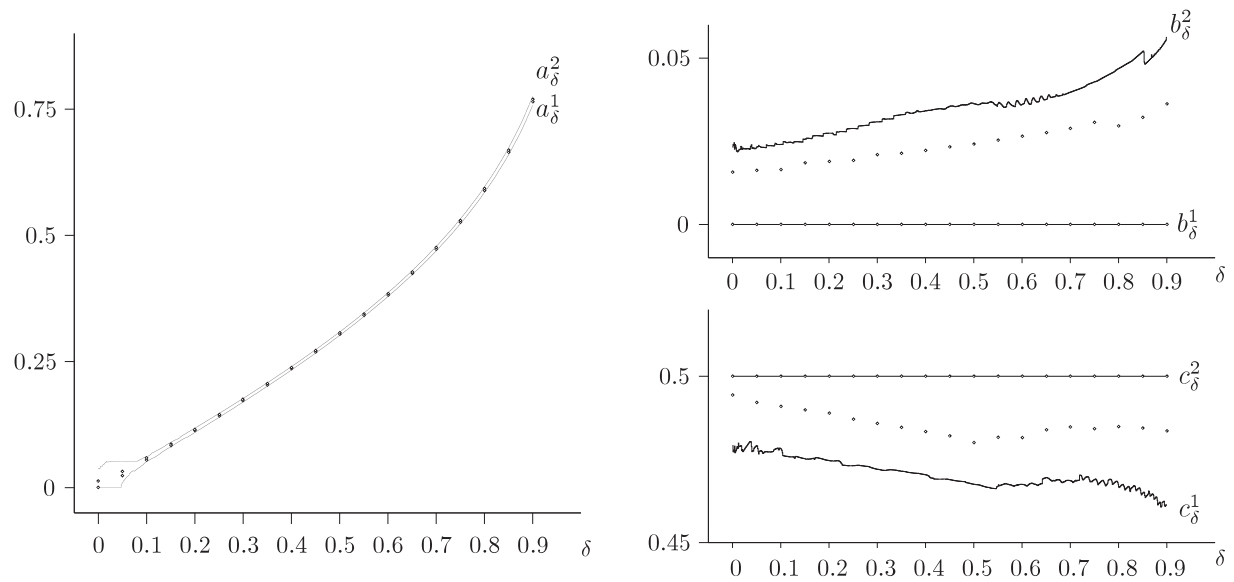

Figure 4 . In each graph, the solid lines represent the enclosures $\left[a_{[\delta]}\right]$, $\left[b_{[\delta]}\right]$ and $\left[c_{[\delta]}\right]$, and the dots represent lower and upper endpoints of $\left[a_{\delta}\right],\left[b_{\delta}\right]$ and $\left[c_{\delta}\right]$ for selected values of $\delta$.

Table 7 lists calculated enclosures $\left[a_{\delta}\right],\left[b_{\delta}\right],\left[c_{\delta}\right]$ and $\left[a_{[\delta]}\right],\left[b_{[\delta]}\right],\left[c_{[\delta]}\right]$ for some selected values of $\delta \in[0,1)$ and intervals $[\delta] \subset[0,1)$, respectively. In Figure 4 the dependence of the enclosures of $a_{\delta}, b_{\delta}$ and $c_{\delta}$ on the ellipticity parameter $\delta$ is displayed.

TABLE 7 . The enclosures $\left[a_{\delta}\right],\left[b_{\delta}\right],\left[c_{\delta}\right]$ and $\left[a_{[\delta]}\right],\left[b_{[\delta]}\right],\left[c_{[\delta]}\right]$ for selected values of $\delta$ and intervals $[\delta]$.

\begin{tabular}{|c|c|c|c|c|c|c|c|}
\hline$\delta$ & {$\left[a_{\delta}\right]$} & {$\left[b_{\delta}\right]$} & {$\left[c_{\delta}\right]$} & {$[\delta]$} & {$\left[a_{[\delta]}\right]$} & {$\left[b_{[\delta]}\right]$} & {$\left[c_{[\delta]}\right]$} \\
\hline 0.00 & $0.0_{0}^{1322}$ & $0.0_{0}^{1574}$ & $0 .{ }_{49437}^{5}$ & $0.0_{0}^{01}$ & $0.0_{0}^{3892}$ & $0.0_{0}^{2323}$ & $0 .{ }_{47917}^{5}$ \\
\hline 0.10 & $0.05_{434}^{842}$ & $0.0_{0}^{1643}$ & $0 .{ }_{49090}^{5}$ & $0.1_{0}^{01}$ & $0.0_{5185}^{6224}$ & $0.0_{0}^{2408}$ & $0 .{ }_{47825}^{5}$ \\
\hline 0.20 & $0.11_{267}^{538}$ & $0.0_{0}^{1893}$ & $0 .{ }_{48883}^{5}$ & $0.2_{0}^{01}$ & $0.1_{0975}^{1834}$ & $0.0_{0}^{2745}$ & $0 .{ }^{5}{ }_{47464}$ \\
\hline 0.30 & $0.17_{202}^{482}$ & $0.0_{0}^{2096}$ & 0.58574 & $0.3_{0}^{01}$ & $0.17_{010}^{726}$ & $0.0_{0}^{3084}$ & $0 .{ }_{47205}^{5}$ \\
\hline 0.40 & $0.23_{523}^{790}$ & $0.0_{0}^{2219}$ & 0.58331 & $0.4_{0}^{01}$ & $0.2_{3350}^{4022}$ & $0.0_{0}^{3415}$ & $0 .{ }_{47039}^{5}$ \\
\hline 0.50 & $0.30_{390}^{661}$ & $0.0_{0}^{2419}$ & $0 .{ }_{48003}^{5}$ & $0.5_{0}^{01}$ & $0.30_{214}^{903}$ & $0.0_{0}^{3631}$ & $0 .{ }_{46752}^{5}$ \\
\hline 0.60 & $0.38_{108}^{398}$ & $0.0_{0}^{2648}$ & $0 .{ }_{48147}^{5}$ & $0.6_{0}^{01}$ & $0.3_{7916}^{8665}$ & $0.0_{0}^{3608}$ & $0 .{ }_{46748}^{5}$ \\
\hline 0.70 & $0.47_{217}^{539}$ & $0.0_{0}^{2882}$ & $0 .{ }_{48468}^{5}$ & $0.7_{0}^{01}$ & $0.4_{6987}^{7862}$ & $0.0_{0}^{3977}$ & $0 .{ }_{46861}^{5}$ \\
\hline 0.80 & $0.5_{8859}^{9239}$ & $0.0_{0}^{2963}$ & $0 .{ }_{48485}^{5}$ & $0.8_{0}^{01}$ & $0.5_{8547}^{9671}$ & $0.0_{0}^{4689}$ & 0.56677 \\
\hline 0.90 & $0.76_{470}^{955}$ & $0.0_{0}^{3619}$ & $0 .{ }_{48352}^{5}$ & $0.9_{0}^{01}$ & $0.7_{5901}^{7719}$ & $0.0_{0}^{6057}$ & $0 .{ }_{46138}^{5}$ \\
\hline
\end{tabular}


REMARK 4.3 .

(1) In [3] it was conjectured that $b_{\delta}=0$ and $c_{\delta}=1 / 2$, that is, that $\sigma\left(\mathfrak{A}_{\delta}\right)$ is of the form

$$
\sigma\left(\mathfrak{A}_{\delta}\right)=i \mathbb{R} \cup \bigcup_{k \in \mathbb{Z}}\left(i k+\left[-a_{\delta}, a_{\delta}\right]\right) .
$$

To prove that this conjecture is false for some $\delta \in[0,1)$, it would be sufficient to show that $\beta_{\delta}^{-} \neq-2$, that is, that $\beta_{\delta}(\rho)<-2$ for some $\rho \in[0, \infty]$ or $\beta_{\delta}(\rho)>-2$ for all $\rho \in[0, \infty]$. In spite of many attempts, no such pair $(\delta, \rho)$ could be determined. This can be interpreted as another numerical indication of the validity of the conjecture.

(2) Although in [15] no concrete numbers for $a_{\delta}$ were stated, at least the graphical results given there (see [15, Figure 4]) seem to correspond very well to the results obtained above.

\section{Second-order problems}

In [1] we calculated guaranteed enclosures of eigenvalues of second-order problems

$$
-y^{\prime \prime}+q y=\lambda y, \quad y(0)=0
$$

on the semi-axis $[0, \infty)$ with complex-valued potentials $q \in L^{1}(0, \infty)$ such that $\lim _{x \rightarrow \infty} q(x)$ $=0$. There we combined a fixed-point theorem with Levinson's asymptotics to deal with the unboundedness of the interval $[0, \infty)$ (see $[\mathbf{1}$, Theorem 3.1]).

In the following, we show that the mere use of Levinson asymptotics (see [1, Theorem 3.3]) already suffices to enclose eigenvalues of (5.1) and that this even improves the enclosures established in $[\mathbf{1}]$.

The Levinson theorem states that for $\lambda \in \mathbb{C} \backslash[0, \infty)$ the $L^{2}$-solution $y_{2}$ of $(5.1)$, suitably normalised, satisfies

$$
\begin{aligned}
& y_{2}(x, \lambda)=\exp (-\sqrt{-\lambda} x)\left(1+\eta_{1}(x)\right), \\
& y_{2}^{\prime}(x, \lambda)=-\sqrt{-\lambda} \exp (-\sqrt{-\lambda} x)\left(1+\eta_{2}(x)\right),
\end{aligned}
$$

where $\Re(\sqrt{-\lambda})>0$ and

$$
\left|\eta_{j}(x)\right| \leqslant \frac{\alpha_{X}}{1-\alpha_{X}} \quad \text { for } j=1,2 \quad \text { with } \alpha_{X}:=\int_{X}^{\infty}|q(s)| d s,
$$

for all $x \geqslant X$ where $X$ is such that $\alpha_{X}<1$. So, if we solve the differential equation $-y^{\prime \prime}+q y=$ $\lambda y$ on $[0, X]$ with the (interval-valued) initial condition

$$
y(X, \lambda)=[E] \exp (-\sqrt{-\lambda} X), \quad y^{\prime}(X, \lambda)=-[E] \sqrt{-\lambda} \exp (-\sqrt{-\lambda} X)
$$

where $[E]$ is an interval such that

$$
\left[1-\frac{\alpha_{X}}{1-\alpha_{X}}, 1+\frac{\alpha_{X}}{1-\alpha_{X}}\right] \subset[E]
$$

and then integrate backwards, it follows that $y_{2}(0, \lambda) \in\left[y_{2}(0, \lambda)\right]$; here $\left[y_{2}(0, \lambda)\right]$ denotes the interval-valued solution of (5.3). Thus, we can apply Lemma 2.1 to the function $y_{2}(0, \cdot)$, using the enclosure $\left[y_{2}(0, \cdot)\right]$, to calculate the number of eigenvalues in a given rectangle $[\lambda]$.

The following two examples illustrate that this direct method by means of Levinson asymptotics can improve, in some cases, the accuracy of the enclosures compared to the method used in $[\mathbf{1}]$. In addition, we were able to increase the length of the cut-off interval $[0, X]$ from $X=10$ to $X=20$ in Example 5.1 and from $X=33$ to $X=50$ in Example 5.2.

Example 5.1 (Squire's problem). The following two sample potentials illustrate the improved accuracy of the algorithm established in this paper. 
- $q(x)=10 i e^{-x}$

$$
\begin{aligned}
\text { fixed-point theorem: } & 2.8122672_{89}^{92}+2.17223818_{78}^{99} i ; \\
\text { direct method: } & 2.812267289948_{29}^{53}+2.172238189004_{20}^{48} i .
\end{aligned}
$$

- $q(x)=100 i \sin (x) e^{-x}$

$$
\begin{aligned}
& \text { fixed-point theorem: } 18.62757_{78}^{82}+12.10064_{69}^{73} i ; \\
& \text { direct method: } 18.6275778679_{68}^{17}+12.100647219_{72}^{83} i
\end{aligned}
$$

EXAmple 5.2 (Resonances of Schrödinger operators). This last example shows that the method developed above allows us to enclose a resonance of the Sturm-Liouville operator induced by

$$
(L y)(x):=-y^{\prime \prime}(x)+x^{2} \exp \left(-0.2 x^{2}\right) y(x), \quad x \in[0, \infty), \quad y(0)=0,
$$

which had previously resisted enclosure by the fixed point method in [1, Example 4.2.3]. As the complex scaling transformation therein contains some typographical errors, we repeat the details below. The transformed differential equation

$$
e^{i \theta}\left(U_{\theta} L U_{\theta}^{-1} y\right)(x)=\lambda y(x), \quad\left(U_{\theta} y\right)(x)=e^{-i \theta / 2} y\left(e^{i \theta / 2} x\right),
$$

is of the form (5.1) with

$$
q(x)=e^{2 i \theta} x^{2} \exp \left(-0.2 e^{i \theta} x^{2}\right), \quad x \in[0, \infty),
$$

and $\lambda$ is an eigenvalue of (5.1) if and only if $e^{-i \theta} \lambda$ is a resonance for (5.4). The direct method of the present paper with $\theta=1.5$ yields the guaranteed enclosure

$$
1.6478_{78}^{81}-12.8293_{18}^{22} i
$$

for a resonance indicated by floating point calculations.

Acknowledgements. The authors would like to thank Professor Nick Trefethen for many valuable comments on an earlier draft of this paper, which have enabled the authors to present a much more modern perspective on this work.

The authors gratefully acknowledge support from the Deutscher Akademischer Austauschdienst (DAAD) and the British Council, grant 313-ARC; the Engineering and Physical Sciences Research Council (EPSRC), grant EP/E037844/1 (M. Langer); and the Deutsche Forschungsgemeinschaft (DFG), grant TR 368/6-2 (C. Tretter).

\section{References}

1. B. M. Brown, M. Langer, M. Marletta, C. Tretter and M. Wagenhofer, 'Eigenvalue bounds for the singular Sturm-Liouville problem with a complex potential', J. Phys. A 36 (2003) 3773-3787.

2. W. K.-H. CHu, 'Spectra of the Orr-Sommerfeld equation: the plane Poiseuille flow for the normal fluid revisited', J. Phys. A 34 (2001) 3389-3392.

3. R. Denk, M. Möller and C. Tretter, 'The spectrum of a parametrized partial differential operator occurring in hydrodynamics', J. London Math. Soc. (2) 65 (2002) 483-492.

4. J. J. Dongarra, B. Straughan and D. W. Walker, 'Chebyshev tau-QZ algorithm methods for calculating spectra of hydrodynamic stability problems', Appl. Numer. Math. 22 (1996) 399-434.

5. P. G. Drazin and W. H. Reid, Hydrodynamic stability (Cambridge University Press, Cambridge, 1982).

6. A. Georgescu, Hydrodynamic stability theory (Martinus Nijhoff Publishers, Dordrecht, 1985).

7. S. K. Godunov, 'Spectral portraits of matrices and criteria of spectrum dichotomy', Computer arithmetic and enclosure methods (Oldenburg, 1991) (North-Holland, Amsterdam, 1992) 25-35.

8. L. Greenberg and M. Marletta, 'Numerical solution of non-self-adjoint Sturm-Liouville problems and related systems', SIAM J. Numer. Anal. 38 (2001) 1800-1845.

9. W. Heisenberg, 'Über Stabilität und Turbulenz von Flüssigkeitsströmen', Ann. Phys. (4) 74 (1924) no. 379, 577-627 (German); 'On stability and turbulence of fluid flows', Tech. Memos. Nat. Comm. Aeronaut. (1951) no. 1291 (English). 
10. D. Hinrichsen and A. J. Pritchard, 'Mathematical systems theory I', Modelling state space analysis and robustness (Springer, Berlin, 2005).

11. D. D. JosePh, 'Eigenvalue bounds for the Orr-Sommerfeld equation', J. Fluid Mech. 33 (1968) 617-621.

12. J. Lahmann and M. Plum, 'On the spectrum of the Orr-Sommerfeld equation on the semiaxis', Math. Nachr. 216 (2000) 145-153.

13. J. Lahmann and M. Plum, 'A computer-assisted instability proof for the Orr-Sommerfeld equation with Blasius profile', Z. Angew. Math. Mech. 84 (2004) 188-204.

14. H. Langer and C. Tretter, 'Spectral properties of the Orr-Sommerfeld problem', Proc. Roy. Soc. Edinburgh Sect. A 127 (1997) 1245-1261.

15. A. Lifschitz, 'Exact description of the spectrum of elliptical vortices in hydrodynamics and magnetohydrodynamics', Phys. Fluids 7 (1995) 1626-1636.

16. C. C. Lin, The theory of hydrodynamic stability (Cambridge University Press, Cambridge, 1955).

17. P. R. NACHTSheim, 'An initial value method for the numerical treatment of the Orr-Sommerfeld equation for the case of plane Poiseuille flow', NASA Technical Report TN D-2414, Lewis Research Center, Cleveland, OH, 1964.

18. N. S. Nedialkov, K. R. Jackson and J. D. Pryce, 'An effective high-order interval method for validating existence and uniqueness of the solution of an IVP for an ODE', Reliab. Comput. 7 (2001) 449-465.

19. B. S. NG and W. H. Reid, 'An initial value method for eigenvalue problems using compound matrices', J. Comput. Phys. 33 (1979) 70-85.

20. S. A. OrszaG, 'Accurate solution of the Orr-Sommerfeld stability equation', J. Fluid Mech. 50 (1971) 689-703.

21. V. A. Romanov, 'Stability of plane-parallel Couette flow', Dokl. Akad. Nauk SSSR 196 (1971) 1049-1051.

22. I. V. Schensted, Contributions to the theory of hydrodynamic stability (University of Michigan, Ann Arbor, 1960).

23. P. J. Schmid and D. S. Henningson, Stability and transition in shear flows (Springer, New York, 2001).

24. J. T. Stuart, R. C. DiPrima, P. M. Eagles and A. Davey, 'On the instability of the flow in a squeeze lubrication film', Proc. R. Soc. Lond. Ser. A 430 (1990) 347-375.

25. L. H. Thomas, 'The stability of plane Poiseuille flow', Phys. Rev. 91 (1953) 780-783.

26. L. N. Trefethen and M. Embree, 'Spectra and pseudospectra', The behavior of nonnormal matrices and operators (Princeton University Press, Princeton, 2005).

27. L. N. Trefethen, A. E. Trefethen, S. C. Reddy and T. A. Driscoll, 'Hydrodynamic stability without eigenvalues', Science 261 (1993) no. 5121, 578-584.

28. Y. Watanabe, M. Plum and M. T. NakaO, 'A computer-assisted instability proof for the Orr-Sommerfeld problem with Poiseuille flow', Z. Angew. Math. Mech. 89 (2009) 5-18.

\author{
B. Malcolm Brown \\ School of Computer Science \\ Cardiff University \\ 5 The Parade, Cardiff CF24 3AA \\ United Kingdom \\ malcolm.brown@cs.cf.ac.uk
}

\author{
Marco Marletta \\ School of Mathematics \\ Cardiff University \\ Senghennydd Road, Cardiff CF24 4AG \\ United Kingdom \\ marco.marletta@cs.cf.ac.uk
}

Markus Wagenhofer
Psylock GmbH

Regerstrasse 4, 93053 Regensburg

Germany

markus.wagenhofer@psylock.com

\author{
Matthias Langer \\ Department of Mathematics and \\ Statistics \\ University of Strathclyde \\ 26 Richmond Street, Glasgow G1 1XH \\ United Kingdom \\ m.langer@strath.ac.uk
Christiane Tretter
Mathematisches Institut
Universität Bern,
Sidlerstrasse 5, 3012 Bern
Switzerland \\ tretter@math.unibe.ch
}

